\title{
Insights From the Lactobacillus johnsonii Genome Suggest the Production of Metabolites With Antibiofilm Activity Against the Pathobiont Candida albicans
}

\author{
Roberto Vazquez-Munoz', Angela Thompson', Jordan T. Russell', Takanori Sobue', \\ Yanjiao Zhou ${ }^{2}$ and Anna Dongari-Bagtzoglou ${ }^{1 *}$ \\ ${ }^{1}$ Department of Periodontology, University of Connecticut Health Center, Farmington, CT, United States, ${ }^{2}$ Department of \\ Psychiatry/Medicine, University of Connecticut Health Center, Farmington, CT, United States
}

\section{OPEN ACCESS}

Edited by:

Harold J. Schreier,

University of Maryland,

Baltimore County, United States

Reviewed by:

Melyssa Negri,

State University of Maringá,

Brazil

Qi-Lin Zhang

Kunming University of Science and

Technology, China

*Correspondence:

Anna Dongari-Bagtzoglou

adongari@uchc.edu

Specialty section:

This article was submitted to

Microbial Physiology and

Metabolism, a section of the

journal Frontiers in Microbiology

Received: 13 January 2022

Accepted: 14 February 2022

Published: 07 March 2022

Citation:

Vazquez-Munoz $R$, Thompson $A$,

Russell JT, Sobue T, Zhou Y and Dongari-Bagtzoglou A (2022) Insights

From the Lactobacillus johnsonii Genome Suggest the Production of Metabolites With Antibiofilm Activity

Against the Pathobiont Candida

albicans.

Front. Microbiol. 13:853762.

doi: 10.3389/fmicb.2022.853762
Lactobacillus johnsonii is a probiotic bacterial species with broad antimicrobial properties; however, its antimicrobial activities against the pathobiont Candida albicans are underexplored. The aim of this study was to study the interactions of $L$. johnsonii with C. albicans and explore mechanisms of bacterial anti-fungal activities based on bacterial genomic characterization coupled with experimental data. We isolated an L. johnsonii strain (MT4) from the oral cavity of mice and characterized its effect on C. albicans growth in the planktonic and biofilm states. We also identified key genetic and phenotypic traits that may be associated with a growth inhibitory activity exhibited against $C$. albicans. We found that $L$. johnsonii MT4 displays $\mathrm{pH}$-dependent and $\mathrm{pH}$-independent antagonistic interactions against $C$. albicans, resulting in inhibition of $C$. albicans planktonic growth and biofilm formation. This antagonism is influenced by nutrient availability and the production of soluble metabolites with anticandidal activity.

Keywords: L. johnsonii, Candida abicans, biofilm, Lactobacillus, bacteriocin, anticandida

\section{INTRODUCTION}

Candida albicans is the most common opportunistic fungal pathogen in immunocompromised hosts (Pfaller and Diekema, 2010; Pfaller et al., 2019). Although it is a member of the mucosal microbiota at different body sites in health, under certain conditions, it can cause invasive mucosal infections (Pendleton et al., 2018). The ability of C. albicans to switch from yeast to hyphal morphotypes and form biofilms increases its virulence on mucosal tissues (Pappas et al., 2018). The biofilm growth form also increases resistance to innate immune effector cells and antimicrobial treatments (Dongari-Bagtzoglou et al., 2009; Vazquez-Munoz et al., 2020).

The clinical outcome of mucosal candidiasis has been adversely affected by the rise of drug-resistant $C$. albicans strains which have become a serious threat to human health (Centers for Disease Control and Prevention, 2019). As the effectiveness of conventional antifungals is diminished, novel strategies are being developed, such as probiotic therapies (Mundula et al., 2019). In this regard, several species from the Lactobacillus complex genus have been studied 
as probiotic therapies in gastrointestinal, vulvovaginal, and oral Candida infections in mouse models and human clinical trials (Vazquez-Munoz and Dongari-Bagtzoglou, 2021). This is due to the fact that certain lactobacilli produce soluble metabolites-i.e., bacteriocins, weak organic acids (such as lactic and acetic acids), and biosurfactants with anticandidal properties (Vazquez-Munoz and Dongari-Bagtzoglou, 2021). Antifungal activities vary across Lactobacillus species and even among strains (Strus et al., 2005; Jang et al., 2019); hence, the studies on relatively unexplored species, such as Lactobacillus johnsonii, and newly isolated strains within this species are novel and can unravel potentially significant probiotic properties.

Recently our group reported that the virulence of $C$. albicans in a mouse model of oropharyngeal candidiasis is attenuated by dietary sucrose. Sucrose significantly enriched the L. johnsonii communities on the oral mucosa during infection with $C$. albicans and caused a reduction in fungal burdens (Bertolini et al., 2021). Since other Lactobacillus species are antagonistic to Candida (Vazquez-Munoz and Dongari-Bagtzoglou, 2021), we hypothesized that $L$. johnsonii might exert a growth inhibitory effect on C. albicans. Lactobacillus johnsonii are Gram-positive, facultatively anaerobic, non-motile bacteria that are part of the $L$. acidophilus group. Like other lactobacilli, L. johnsonii is considered as a GRAS (generally recognized as safe) microorganism and is regarded as a probiotic (Marcial et al., 2017; Zheng et al., 2020). Lactobacillus johnsonii is a member of the human health-associated gastrointestinal and vaginal mucosal microbiota, two sites afflicted by mucosal candidiasis (Fujisawa et al., 1992; Assefa et al., 2015; Zheng et al., 2020). However, there is scant information on the interactions between L. johnsonii and C. albicans, and the limited available information is contradictory (Gil et al., 2010; Assefa et al., 2015; Eryilmaz et al., 2019).

We recently isolated $L$. johnsonii strain MT4 from the oral mucosa of mice receiving a sucrose-enriched diet. This strain was identified as $L$. johnsonii via whole $16 \mathrm{~S}$ rRNA gene sequencing (Bertolini et al., 2021). Human clinical trials suggest that $L$. johnsonii-based probiotics may reduce the burden of certain infections and metabolic disorders (Cruchet et al., 2003). In this work, our aim was to functionally characterize the genome of strain MT4, assess its effects on the planktonic and biofilm growth of the pathobiont C. albicans, and explore relevant mechanisms of antifungal activity. We discovered that L. johnsonii MT4 displays $\mathrm{pH}$-dependent and $\mathrm{pH}$-independent anticandidal properties, mediated by the release of soluble metabolites, resulting in inhibition of C. albicans planktonic growth and biofilm formation. Our results also shed new light on existing contradictory data regarding the impact of lactic acid-mediated acidification on C. albicans growth.

\section{MATERIALS AND METHODS}

\section{Strains}

Lactobacillus johnsonii strain MT4 was reactivated from frozen stocks in $5 \mathrm{ml}$ MRS broth (Difco ${ }^{\mathrm{TM}}$ ) in an anaerobic chamber at $37^{\circ} \mathrm{C}$ overnight. Candida albicans SC5314 (ATCC MYA-2876), also reactivated from frozen stocks, was sub-cultured in YPD broth (Yeast Extract, Sigma ${ }^{\circledR}$; Bacto ${ }^{\mathrm{TM}}$ Peptone, Gibco; and Dextrose, J.T.Baker ${ }^{\circledR}$ ), and incubated aerobically at $30^{\circ} \mathrm{C}$ in an orbital shaker, overnight. Bacterial and fungal overnight cultures were washed in PBS and adjusted to their final concentration in either MRS, MRS w/o dextrose (USbiological), BHI (BBL ${ }^{\mathrm{TM}}$, $\mathrm{BD}$ ), or biofilm medium (RPMI medium 1640 [Gibco] supplemented with $10 \%$ BHI, and 10\% Fetal Bovine Serum [Gibco]), as described below. In some experiments, L. johnsonii type strain ATCC 33200 was used for comparison.

\section{Genomic Characterization of Lactobacillus johnsonii MT4 Strain}

Genome sequencing, taxonomy, and comparative genomics of L. johnsonii MT4 strain were assessed as follows: (I) Sequencing: DNA was extracted using the DNeasy ${ }^{\circledR}$ Blood \& Tissue Kit (Qiagen, United States; Bertolini et al., 2021). The extracted genomic DNA was assessed for concentration and size using the Qubit 3.0 HS dsDNA assay (Life Technologies, Carlsbad, CA, United States) and the Tapestation 4200 genomic DNA assay (Agilent Technologies, Santa Clara, CA, United States), respectively. The DNA sample was diluted to $0.2 \mathrm{ng} / \mu \mathrm{l}$, and the sequencing library was prepared using the Illumina Nextera XT DNA kit (Illumina, San Diego, CA, United States) according to the manufacturer's instructions. The library was again checked for concentration and size ( $450 \mathrm{bp}$ average library length; average insert size of $315 \mathrm{bp}$ ) as before, and the sequencing library was prepared using the Illumina Nextera XT DNA kit (Illumina, San Diego, CA, United States) and sequenced using $2 \times 150$ bp format on an Illumina NovaSeq 6000 at the Center for Genome Innovation (Institute for Systems Genomics, University of Connecticut). Reads pertaining to $1 \%$ PhiX control spike-in were filtered and removed via USEARCH v11.0.667. The Whole Genome Sequence (WGS) of MT4 was assembled using Unicycler v0.4.8, which utilizes the SPAdes assembler v3.15.2 (Bankevich et al., 2012), at the University of Connecticut's Xanadu HighPerformance Computing Cluster; (II) Taxonomy: the contigs resulting from the assembly of MT4 were taxonomically classified using Kraken2 v2.0.8-beta (Wood et al., 2019). Phylogenetic analysis on core genes was performed to find the closest $L$. johnsonii MT4 strain relatives. Seventeen whole-Genome Sequences of $L$. johnsonii strains from the NCBI database (strains NCK2677, ATCC 33200, N6.2, 3DG, BS15, Byun_jo_01, DC22.2, DPC_6026, FI9785, G2A, GHZ10a, IDCC9203, NCC_533, UMNLJ21, UMNLJ22, ZLJ010, and pf01) were compared. The assembled MT4 genome and the NCBI genomes were annotated with Prokka v1.14.6 (Seemann, 2014), and the comparative genomics analysis was performed with Roary v3.13.0 (Page et al., 2015) to identify genes unique and in common between our MT4 isolate and the other 17 NCBI genomes of $L$. johnsonii strains. Roary generates an alignment of the core genes using PRANK (Löytynoja, 2014). Phylogenetic analysis of the L. johnsonii strains was done using SeaView v5.0.4 (Gouy et al., 2010). The core gene alignment was curated for further analysis using GBlocks (Castresana, 2000) to remove poorly aligned regions, including large gaps. The phylogeny 
was generated with PhyML (Guindon et al., 2010) using the GTR DNA substitution model, which was determined as the optimal model using the Smart Model Selection (SMS) tool web server (Lefort et al., 2017) and branch support values calculated using the aLRT method. ${ }^{1}$ Nucleotide equilibrium frequencies, invariable sites, and across-site rate variation were set to "optimized," with the BioNJ option selected (Gascuel, 1997). Finally, SeaView uses the PHYLIP package (Felsenstein, 1993) for tree parsimony. The presence or absence of genes and proteins in MT4 was also verified with searches using BLAST (Altschul et al., 1990) and tblastn, respectively.

\section{Phenotypic Characterization of Lactobacillus johnsonii Growth on Different Culture Media}

Lactobacillus johnsonii was grown on MRS (BD), BHI (BD), YPD, KSFM (Gibco), and F-12 (Gibco). Lactobacilli were incubated static, aerobically with $5 \% \mathrm{CO}_{2}$, at $37^{\circ} \mathrm{C}$. Optical density $(\lambda=600 \mathrm{~nm})$ was measured every $90 \mathrm{~min}$. The $\mathrm{pH}$ of growth media was measured at $t=24 \mathrm{~h}$.

\section{Aggregation}

Lactobacillus johnsonii MT4 auto-aggregation phenotype was assessed in MRS broth after growth under aerobic conditions with $5 \% \mathrm{CO}_{2}$, at $37^{\circ} \mathrm{C}$, for $24 \mathrm{~h}$. Auto-aggregation phenotype was defined as positive if the overnight cultures settled at the bottom with no turbidity and negative if they showed turbidity (Jankovic et al., 2003). The non-aggregating L. johnsonii ATCC 33200 strain was used as a negative control.

\section{Assessment of D/L-Lactate Production}

The DL-lactate kit (Megazyme) was used following the manufacturer's recommendations with minor changes. Briefly, supernatants from overnight $L$. johnsonii and C. albicans in single-or dual-species cultures were deproteinized with ice-cold $1 \mathrm{M}$ hydrochloric acid $1 \mathrm{M} \mathrm{NaOH}$, and $20 \mu \mathrm{l}$ of each sample was tested in duplicate. Standard curves were prepared in the corresponding culture medium. Absorbance was measured at $\lambda=340 \mathrm{~nm}$, and D-/L-lactate concentrations were calculated in two independent experiments.

\section{Effect of Lactobacillus johnsonii on Candida albicans Planktonic Growth}

C. albicans $\left(5 \times 10^{4}\right.$ cells $\left.\mathrm{ml}^{-1}\right)$ and $L$. johnsonii $\left(5 \times 10^{4}\right.$ to $5 \times 10^{8}$ cells $\mathrm{ml}^{-1}$ ) were cocultured in MRS or BHI broth aerobically with $5 \% \mathrm{CO}_{2}$ for $24 \mathrm{~h}$, at $37^{\circ} \mathrm{C}$. These growth conditions allow planktonic growth of $C$. albicans exclusively in the yeast form. The influence of media acidification on C. albicans growth was assessed in lactic acid-(DL-LA, Sigma-Aldrich)-supplemented MRS broth ( $\mathrm{pH} 4.0 \pm 0.05,142 \mathrm{mM}$ ) or BHI broth ( $\mathrm{pH} 5.5 \pm 0.08$, $28.3 \mathrm{mM}$ ). To assess the influence of carbohydrate availability, single- or dual-species cultures were tested in MRS broth without dextrose (MRSm) or with added carbohydrates

${ }^{1} \mathrm{http}: / /$ www.atgc-montpellier.fr/sms/
( $2 \%$ sucrose or $2 \%$ dextrose). At the end of each culture period, L. johnsonii viable counts (CFU) were estimated by plating serially 10 -fold diluted cultures onto MRS agar plates, incubated anaerobically at $37^{\circ} \mathrm{C}$, for $48 \mathrm{~h}$. C. albicans yeast cell numbers were assessed by counting in a Neubauer chamber after fixation in $1 \%$ paraformaldehyde (PFA, Sigma).

\section{Preparation of Non-viable Lactobacillus johnsonii}

Some studies have reported that exopolysaccharides from the cell wall of UV-inactivated bacterial cells may decrease the burden of Candida infections (Wagner et al., 2000) and reduce the dimorphic transition (Allonsius et al., 2019). Thus, the impact of inactivated L. johnsonii cells on $C$. albicans was evaluated. Lactobacillus johnsonii cells were UV-light-inactivated in a UVP crosslinker instrument (Analytik Jena; $254 \mathrm{~nm}, 1,500 \mathrm{~mJ} \mathrm{~cm}^{-2}$, at $8 \mathrm{~cm}$ from the UV lamp) for 12 cycles; Heat-killed organisms were prepared at $95^{\circ} \mathrm{C}$ for $30 \mathrm{~min}$ on a heat block (VWR). To verify that bacteria were killed, $10 \mu \mathrm{l}$ from each suspension were transferred into $1 \mathrm{ml}$ of MRS and incubated at $37^{\circ} \mathrm{C}$ for $48 \mathrm{~h}$. Candida albicans $\left(\sim 5 \times 10^{4}\right.$ cells $\left.\mathrm{ml}^{-1}\right)$ with killed lactobacilli $\left(\sim 5 \times 10^{6}\right.$ cells $\mathrm{ml}^{-1}$ ) were suspended in BHI broth and were incubated as described above.

\section{Preparation of Cell-Free Supernatants}

Supernatants from L. johnsonii single-species cultures (Lj-cellfree supernatant (CFS), starting at $5 \times 10^{7} \mathrm{cell} \mathrm{ml}^{-1}$ ) or in cocultures with Candida albicans (CC-CFS, starting at $5 \times 10^{4}$ cell $\mathrm{ml}^{-1}$ ) were prepared in MRS broth, BHI broth, or biofilm medium under aerobic conditions with $5 \% \mathrm{CO}_{2}$, at $37^{\circ} \mathrm{C}$, for $24 \mathrm{~h}$. CFS were collected by centrifugation at $4,000 \mathrm{rpm}$ for $20 \mathrm{~min}$. The $\mathrm{pH}$ of supernatants was measured, and each supernatant was divided into two aliquots; one was kept at the original $\mathrm{pH}$ (acidic-CFS, $\mathrm{pH} 3.87 \pm 0.03$ for MRS broth, $5.40 \pm 0.04$ for $\mathrm{BH}$, and $\mathrm{pH} 6.08 \pm 0.18$ for biofilm medium), while the other was $\mathrm{pH}$-adjusted with a $1 \mathrm{M} \mathrm{NaOH}$ solution (neutralized-CFS, pH $6.6 \pm 0.14$ for MRS, pH 7.4 \pm 0.06 for BHI, and $\mathrm{pH} 8.68 \pm 0.04$ for biofilm medium). CFS were sterile filtered using a $0.2 \mu \mathrm{m}$ filter (Corning). CFS were stored at $4^{\circ} \mathrm{C}$. The effect of CFS on C. albicans $\left(\sim 5 \times 10^{4}\right.$ cells $\left.\mathrm{ml}^{-1}\right)$ was tested in growth media supplemented with $50 \%$ CFS or PBS as control.

\section{Impact of Lactobacillus johnsonii on Candida albicans Biofilm Growth} Biofilm Growth

Lactobacillus johnsonii $\left(5 \times 10^{5}\right.$ to $5 \times 10^{7}$ cells $\left.\mathrm{ml}^{-1}\right)$ and C. albicans $\left(5 \times 10^{4}\right.$ cells $\left.\mathrm{ml}^{-1}\right)$ were suspended in biofilm medium (80\% RPMI, 10\% BHI, and 10\% FBS) and seeded in multiwell plates or into $\mu$-Slide 8 -well chambered slides (IBIDI GmbH, Gräfelfing, Germany) and incubated statically, aerobically with $5 \% \mathrm{CO}_{2}$ at $37^{\circ} \mathrm{C}$ for up to $48 \mathrm{~h}$. Singlespecies cultures were used as control. To visualize L. johnsonii, bacteria were stained with $1 \mathrm{mM}$ CellTracker ${ }^{\mathrm{TM}}$ Deep Red dye (Thermo Fisher Scientific). Fungal cells were stained 
with Calcofluor White for $10 \mathrm{~min}$, washed in PBS, and fixed with $4 \%$ paraformaldehyde (PFA). The impact of physical contact between the lactobacilli and Candida on fungal biofilm growth was assessed by seeding L. johnsonii $\left(5 \times 10^{6}\right.$ cell ml $\left.{ }^{-1}\right)$ into a Millicell ${ }^{\circledR} 0.4 \mu \mathrm{m}$ PCF Cell Culture Insert (Millipore, United States). The inserts were placed into the wells of 24-well plates (Corning) containing C. albicans $\left(5 \times 10^{4}\right.$ cell $\left.\mathrm{ml}^{-1}\right)$. Biofilms were grown for $24 \mathrm{~h}$, as above. Exclusion and displacement experiments were performed to assess the effect of preformed biofilms on the ability of the other microbial species to form a biofilm. The supernatant was removed, and preformed biofilms were washed twice with PBS. In exclusion assays, C. albicans $\left(5 \times 10^{4}\right.$ cells $\left.\mathrm{ml}^{-1}\right)$ suspended in fresh biofilm medium was transferred to preformed $L$. johnsonii biofilms. In displacement assays, L. johnsonii $\left(5 \times 10^{7}\right.$ cells $\mathrm{ml}^{-1}$ ) suspended in fresh medium was added to preformed C. albicans biofilms. For negative controls, cell-free medium was added to the single-species $24 \mathrm{~h}$-old preformed biofilms. Plates were incubated for an additional $24 \mathrm{~h}$, as described above.

\section{Biofilm Analyses}

\section{Biovolumes and Thickness}

Micrographs were obtained at a $\times 400$ magnification in a Microscope (Zeiss), with the DAPI $\left(\lambda_{\mathrm{exc}}=358 \mathrm{~nm}, \lambda_{\mathrm{em}}=463 \mathrm{~nm}\right)$ and CY3 $\left(\lambda_{\text {exc }}=549 \mathrm{~nm}, \lambda_{\text {em }}=562 \mathrm{~nm}\right)$ fluorescence channels and using the Z-stack mode. Images were post-processed in Zen Blue (v.3.0, Zeiss) and analyzed with IMARIS Cell Imaging Software (Oxford Instruments) using the Create Surface tool to reconstruct $3 \mathrm{D}$ images from the biofilms and assess their volumes.

\section{Biomass}

Fungal biomass was quantified by qPCR. DNA from biofilms grown on 24-multiwell plates was extracted using the Yeast DNA Extraction Kit (Thermo Scientific), following the manufacturer's recommendations. A region from the fungal rRNA operon was amplified using the primers 5.8S GTGAATCATCGARTC TTTGAAC (forward primer) and 28S-1 TATGCTTAAGTT CAGCGGGTA (reverse primer) under the qPCR conditions reported by Khot et al. (2009). Fungal biomass was directly correlated to the number of amplicons. Each experiment contained untreated biofilms and cell-free media as controls.

\section{Metabolic Activity}

The fungal metabolic activity was assessed via XTT as described elsewhere (Pierce et al., 2008). Briefly, biofilms were incubated for $30 \mathrm{~min}$ with Penicillin G/Streptomycin $\left(100 \mu \mathrm{g} \mathrm{ml}^{-1}\right.$, Gibco) to remove the metabolic signal from lactobacilli. Then, biofilms were washed with BPS, XTT/menadione was added, and samples were incubated for $2.5 \mathrm{~h}$. Absorbance was read at $490 \mathrm{~nm}$.

\section{Reproducibility and Statistical Analyses}

Data from at least two independent experiments with technical replicates were analyzed for statistical significance using One-Way ANOVA with Kruskal-Wallis posttest in Prism v9.2.0 (GraphPad Software, LLC).

\section{RESULTS}

\section{Genomic Characterization of Lactobacillus johnsonii Strain MT4}

The MT4 genome assembly resulted in 68 contigs with an N50 of $90.96 \mathrm{~kb}$. All but 1 contig were classified as $L$. johnsonii, with a single contig classified as L. crispatus based on the Kraken2 results (Supplementary Table 1). The 1,883,026bp genome has a GC content of $34.4 \%$. In total, 1,865 genes were predicted, including 1,772 protein-coding genes and 93 RNA genes (34 miscellaneous/non-coding RNA, 3 rRNA, 55 tRNA, and one tmRNA). Phylogenetic analysis on core genes shows the relation of this strain to 17 Lactobacillus johnsonii strains deposited in the NCBI database (Figure 1), indicating that strain MT4 is almost identical to strain NCK2677, also isolated from the GI

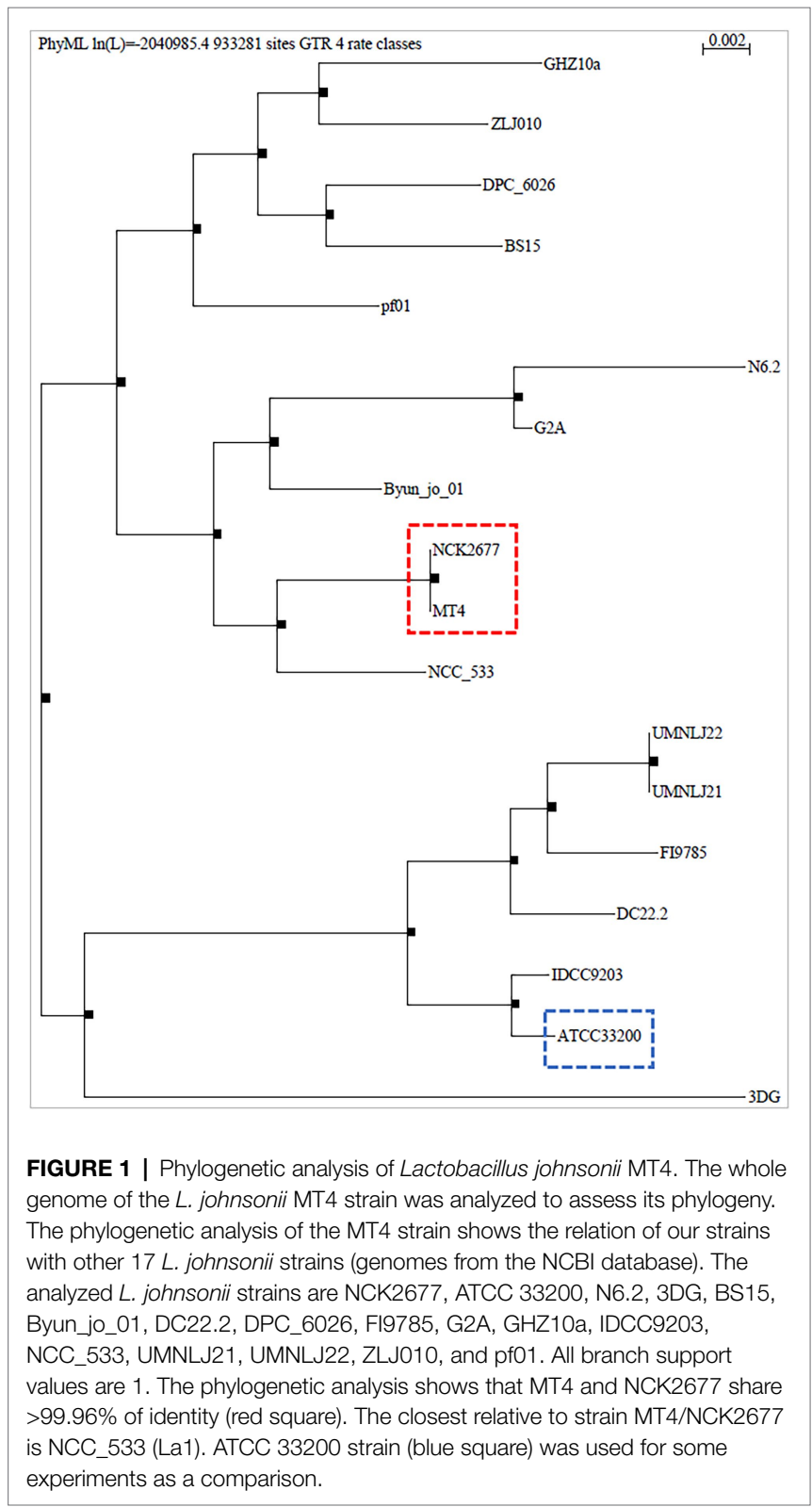


tract of C57BL/6 mice (O'Flaherty et al., 2020), sharing more than $99.96 \%$ identity of their genomes. The closest relative to the MT4/NCK2677 strains is NCC 533 (La1), a strain with probiotic properties (Yamano et al., 2006). Functional genomics analysis revealed that strain MT4 possesses genes encoding products that are similar to reported anticandidal metabolites (a bacteriocin, two hydrolases, and a biosurfactant; Table 1). The alignment analysis is listed in Supplementary Table 2. MT4 also possesses genes for both $\mathrm{D}$ - and L-Lactate synthesis and other metabolites of interest, such as the bacteriocins lactacin-F and helveticin J, and a glucanase (glycoside hydrolase family 8 ).

TABLE 1 | Lactobacillus johnsonii strain MT4 possesses genes encoding for metabolites similar to products reported to display anticandidal activity.

\begin{tabular}{|c|c|c|c|}
\hline Metabolite & Type & $\begin{array}{l}\text { Reported } \\
\text { anticandidal effect }\end{array}$ & References \\
\hline Bacillomycin D & $\begin{array}{l}\text { Lipopeptide, } \\
\text { Bacteriocin }\end{array}$ & $\begin{array}{l}\text { Disruption of the fungal } \\
\text { cell membrane by } \\
\text { forming ion-conducting } \\
\text { pores. } \\
\text { Bacillomycin D-like } \\
\text { peptides inhibit the } \\
\beta-1,3-\text { glucan synthesis. }\end{array}$ & $\begin{array}{l}\text { Olfa et al., 2015; } \\
\text { Hajare et al., } \\
2016\end{array}$ \\
\hline Surfactin & $\begin{array}{l}\text { Cyclo-lipopeptide } \\
\text { biosurfactant }\end{array}$ & $\begin{array}{l}\text { Prevent fungal } \\
\text { adhesion to surfaces, } \\
\text { reducing biofilm } \\
\text { formation }\end{array}$ & $\begin{array}{l}\text { Nelson et al., } \\
2020\end{array}$ \\
\hline Glucanase & $\begin{array}{l}\text { Glycoside } \\
\text { hydrolase family } 8\end{array}$ & $\begin{array}{l}\text { Hydrolysis of } \\
\text { O-glycosyl compounds } \\
\text { from Candida cell wall. }\end{array}$ & Jung, 2018 \\
\hline Msp1/p75 & $\begin{array}{l}\text { Peptidoglycan } \\
\text { hydrolase }\end{array}$ & $\begin{array}{l}\text { Chitinase activity; } \\
\text { degrades Candida cell } \\
\text { wall and inhibits hyphal } \\
\text { morphogenesis }\end{array}$ & $\begin{array}{l}\text { Allonsius et al., } \\
2019\end{array}$ \\
\hline
\end{tabular}

\section{Phenotypic Traits of Lactobacillus johnsonii MT4 \\ Growth on Different Media}

Lactobacillus johnsonii MT4 displayed the best aerobic growth rate on MRS broth, followed by BHI broth, while it displayed poor growth in all other media (Supplementary Figure 1A).

\section{Auto- and Co-aggregation}

Overnight cultures of $L$. johnsonii strain MT4 showed a partial degree of auto-aggregation, whereas the strain ATCC 33200 did not (Supplementary Figure 1B). Additionally, L. johnsonii MT4 co-aggregated with C. albicans cells in dual-species biofilms, particularly on hyphae (Figure 2).

\section{Acidification}

Growth of L. johnsonii MT4 in MRS, BHI, and biofilm medium (80\% RPMI, 10\% BHI, 10\% FBS) displayed a different degree of acidification in overnight cultures (Figure 3, bottom table). Regardless of the starting inoculum size $\left(10^{4}-10^{7}\right.$ cell $\mathrm{ml}^{-1}$ range), lactobacilli acidified MRS broth to $\mathrm{pH} 3.9$ (from 6.5), BHI to $\mathrm{pH} 5.5$ (from 7.5), and biofilm medium to $\mathrm{pH} 6$ (from 8.4). In addition to having different starting $\mathrm{pH}$, the difference in $\mathrm{pH}$ at the end of the growth period in the two media may be associated with a different buffering capacity or the production of different amounts of organic acids in each medium. C. albicans had a small neutralizing effect on the $\mathrm{pH}$ of the growth media in dual-species cultures with L. johnsonii in BHI and biofilm medium only (Figure 3).

\section{DL-Lactate Production}

Lactobacillus johnsonii is reported to produce both the D and L enantiomers of lactate (Fujisawa et al., 1992). The functional genome analysis and a DL-lactate assay confirmed that strain
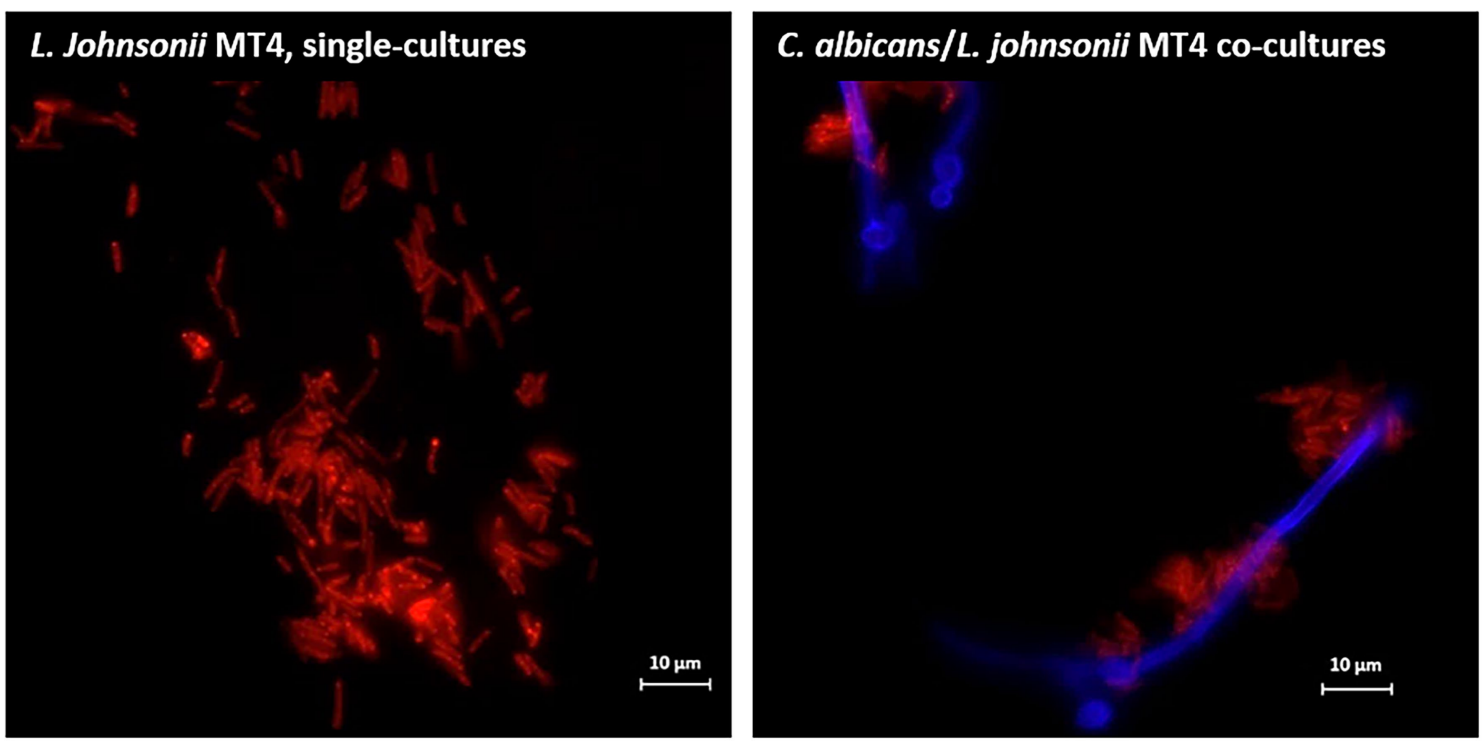

FIGURE 2 | Lactobacillus johnsonii co-aggregates with Candida albicans cells. L. johnsonii (red) auto-aggregates and co-aggregates with C. albicans (blue). The yeast and the lactobacilli were grown on biofilm media for $3 \mathrm{~h}$. Lactobacilli were found in physical proximity with Candida cells, particularly along the hyphae. Scale bar $=10 \mu \mathrm{m}$. 


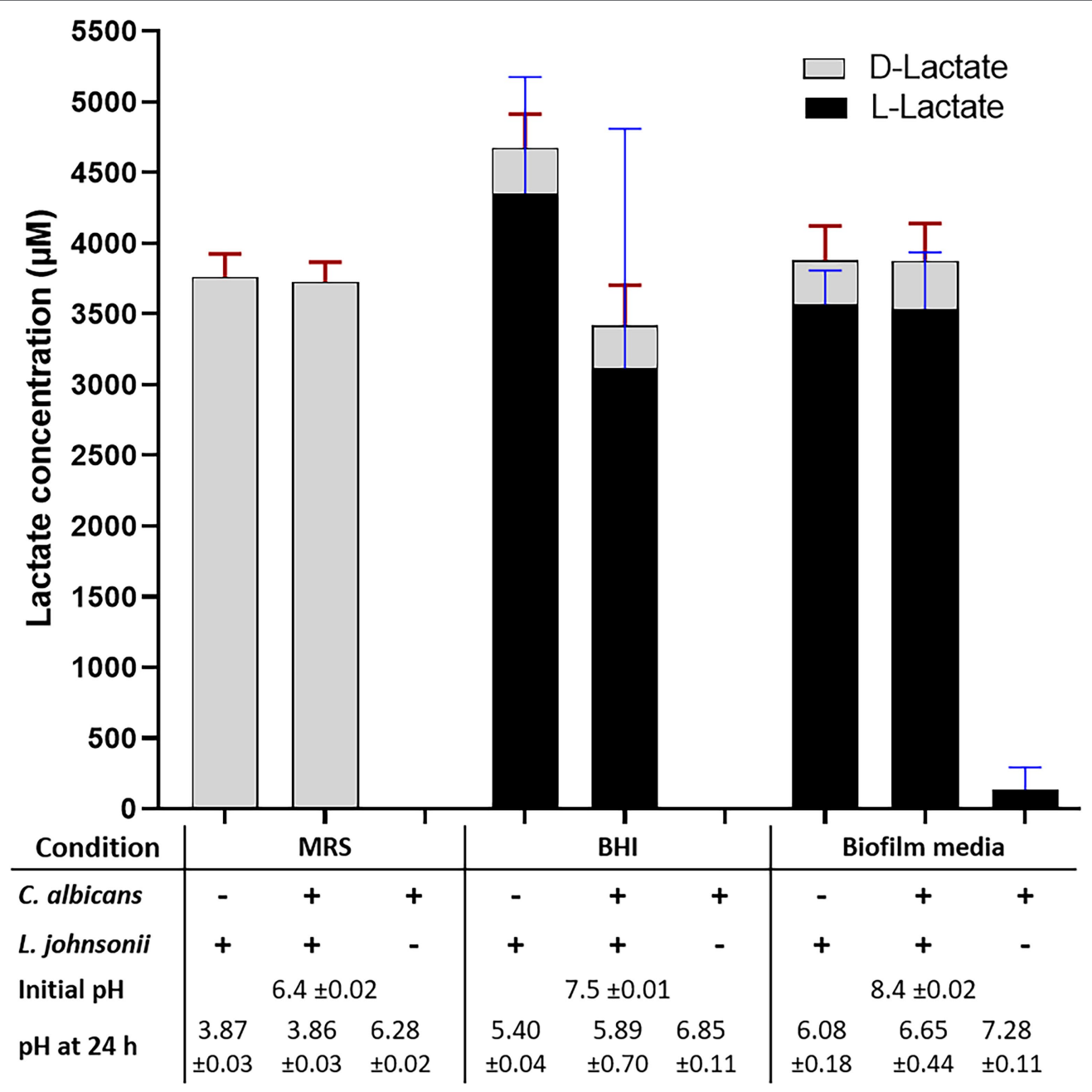

FIGURE 3 | Lactate production and pH on different culture media. The production of DL-lactate by the MT4 strain was measured in various growth media. The growth media influence DL-Lactate production and ratio. In MRS, the D enantiomer is favored, while the L enantiomer production takes over in BHI and biofilm media. Acidification of the culture media does not correspond to the total DL-lactic acid concentration, which may be due to the particular buffering capacity of each growth media and the presence of other organic acids.

MT4 produces both enantiomers (Figure 3). Interestingly, the type of growth medium influenced the D-/L-lactate ratio; D-lactate was favored in MRS broth with both MT4 (Figure 3) and ATCC 33200 (Supplementary Figure 2A) strains, whereas L-lactate was predominant in. BHI broth and biofilm medium, representing $93 \%$ and $92 \%$ of the total production, respectively. The total DL-lactate production was similar in all growth media, ranging between 3.5-4.5 mM (Figure 3). Candida albicans lactate production was negligible and did not influence the lactate production of L. johnsonii (Figure 3).

\section{Lactobacillus johnsonii Inhibits Candida albicans Planktonic Growth}

We first tested the effect of L. johnsonii MT4 on C. albicans planktonic growth in the two media in which this strain showed the best growth. In both MRS and BHI broth L. johnsonii inhibited $C$. albicans growth following a dose-repose trend (Figures 4A,B). The inhibition of C. albicans growth was more pronounced in MRS, which also showed higher acidification at the end of the coculture period (MRS pH 3.9 vs. BHI pH 5.5). Lactobacillus johnsonii ATCC 33200 displayed similar anticandidal activity in MRS broth (Supplementary Figure 2B).

To assess the role of acidification in inhibiting fungal growth, we supplemented the media with sufficient amounts of lactic acid to lower the $\mathrm{pH}$ to levels comparable with a $24 \mathrm{~h}$ culture of L. johnsonii. Candida albicans growth inhibition in lactic acid-supplemented MRS broth was similar to L. johnsonii-induced inhibition, showing that lactic acid-induced acidic $\mathrm{pH}$ alone is sufficient to cause growth inhibition in this medium. In contrast, growth inhibition in lactic-acid supplemented BHI was significantly lower than that induced by live bacteria suggesting that acidic $\mathrm{pH}$ alone is not responsible for the growth inhibition observed in this medium (Supplementary Figure 3). 

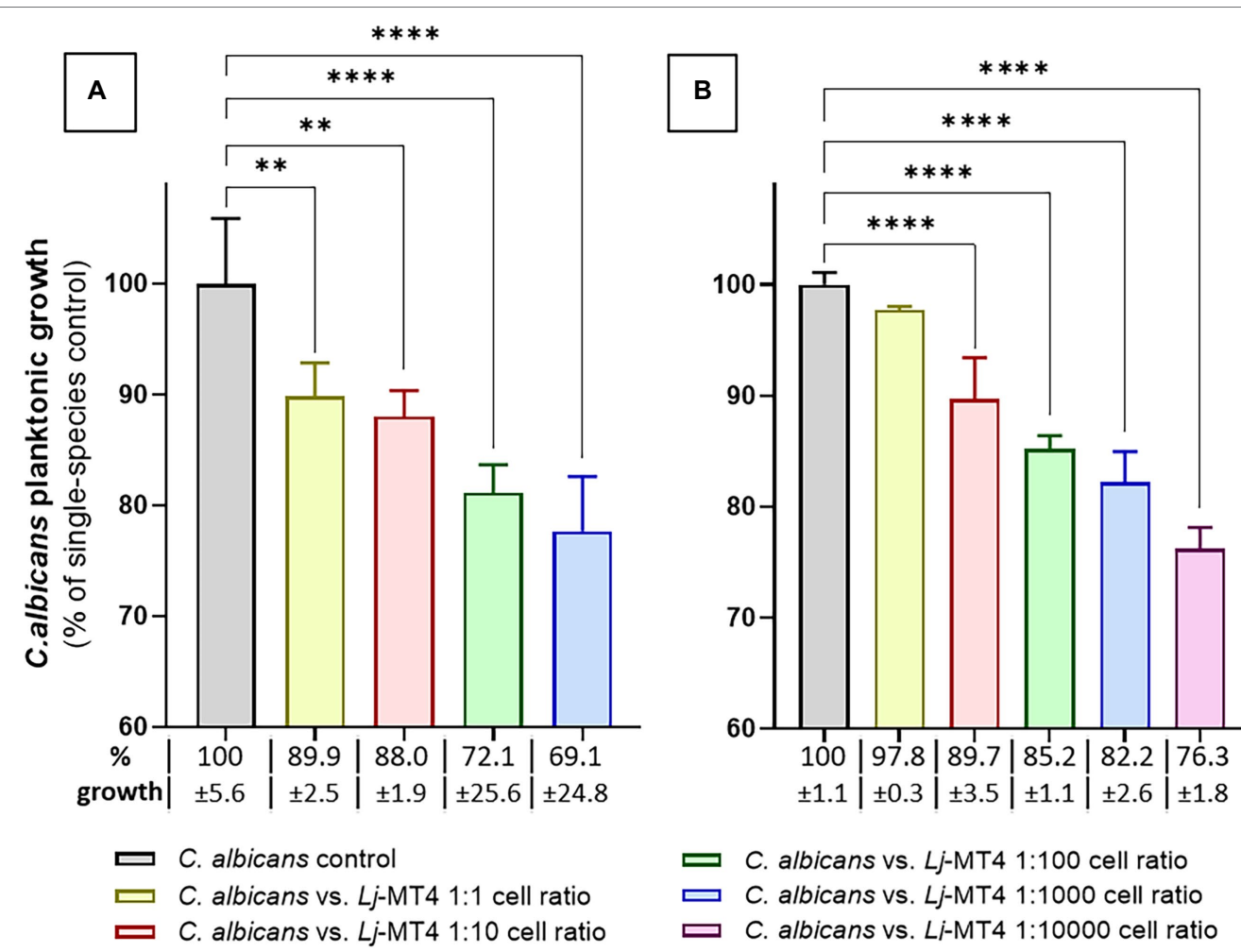

FIGURE 4 | Lactobacillus johnsonii inhibits C. albicans planktonic growth. The effect of L. johnsonii MT4 (Lj) on the planktonic growth of C. albicans (Ca) was assessed in two different growth media. L. johnsonii at different starting concentrations-inhibited C. albicans planktonic growth in a dose-response pattern. (A) MRS broth, and (B) BHI broth. Candida albicans growth inhibition is higher on MRS. One-Way ANOVA, Dunnett posttest, ${ }^{* *} p \leq 0.01$ and ${ }^{* * * *} p \leq 0.0001$. In all dual-species cultures, MRS was acidified to $\mathrm{pH} \sim 3.9$ and $\mathrm{BHI}$ to $\mathrm{pH} \sim 5.5$. 100\% growth corresponds to $8.16 \pm 0.41 \mathrm{SD}$ and $7.47 \pm 0.08 \mathrm{SD}$ yeast cells $\mathrm{ml}^{-1}$ (average, log 10 values) in MRS and $\mathrm{BH}$ broth, respectively.

\section{Carbohydrate Availability Plays a Role in Candida albicans Growth Inhibition in MRS Broth}

We next evaluated the impact of carbohydrate availability on Lactobacillus fitness and its ability to inhibit Candida growth in MRS broth. In carbohydrate-free MRSm, there was a significantly reduced L. johnsonii growth rate (Supplementary Figure 4), lactate production was significantly curtailed, and the $\mathrm{pH}$ of the growth media remained close to the initial $\mathrm{pH}$ (Figure 5A). As expected, supplementing the media with either $2 \%$ dextrose or $2 \%$ sucrose fully restored lactate production and caused media acidification, while co-culture with C. albicans did not affect the amounts of lactate produced (Figure 5A). In MRS broth, growth inhibition of C. albicans by $L$. johnsonii MT4 required the availability of carbohydrates (Figure 5B), being higher in the $2 \%$ dextrosesupplemented MRSm compared to the non-supplemented or $2 \%$ sucrose-supplemented MRSm. Since the Candida-inhibitory effect of L. johnsonii was significantly curtailed when bacterial carbohydrate metabolism was suppressed in MRSm, we hypothesized that bacterial viability is also required for anticandidal activity. As expected, neither UV- nor heat-killed L. johnsonii inhibited C. albicans yeast growth in MRS broth (Figure 6A). Collectively these results indicate that carbohydrate availability influences the ability of $L$. johnsonii to produce weak organic acids and metabolites with anticandidal properties that may be responsible for the growth inhibition of the yeast in MRS.

To further dissect the role of soluble metabolites released in culture media on the growth inhibition observed in MRS broth, we tested the effect of CFS (i.e., Lactobacillus spent media) on C. albicans growth. CFS from Lactobacillus cultures in MRS broth showed significant anticandidal activity, similar to co-cultures with live lactobacilli or lactic acid-acidified media (Figures 6A,B). However, the $\mathrm{pH}$-adjusted $\mathrm{CFS}(\mathrm{pH} \sim 6.5)$ from Lactobacillus cultures in MRS broth had significantly reduced anticandidal activity, suggesting that acidic $\mathrm{pH}$ is required for most anticandidal activity of Lactobacillus metabolites in MRS broth. Similar results were obtained with CFS prepared from cocultures of C. albicans and L. johnsonii (Figure 6B).

\section{Lactobacillus johnsonii Reduces the Ability of Candida albicans to Form Biofilms}

Single species C. albicans biofilms uniformly covered the well surfaces (Figure 7A), while L. johnsonii MT4 biofilms consisted of scattered clusters of cells (Figure 7B). Lactobacillus johnsonii MT4 reduced the metabolic activity of $C$. albicans biofilms in a dose-response pattern (Supplementary Figure 5). The ability 


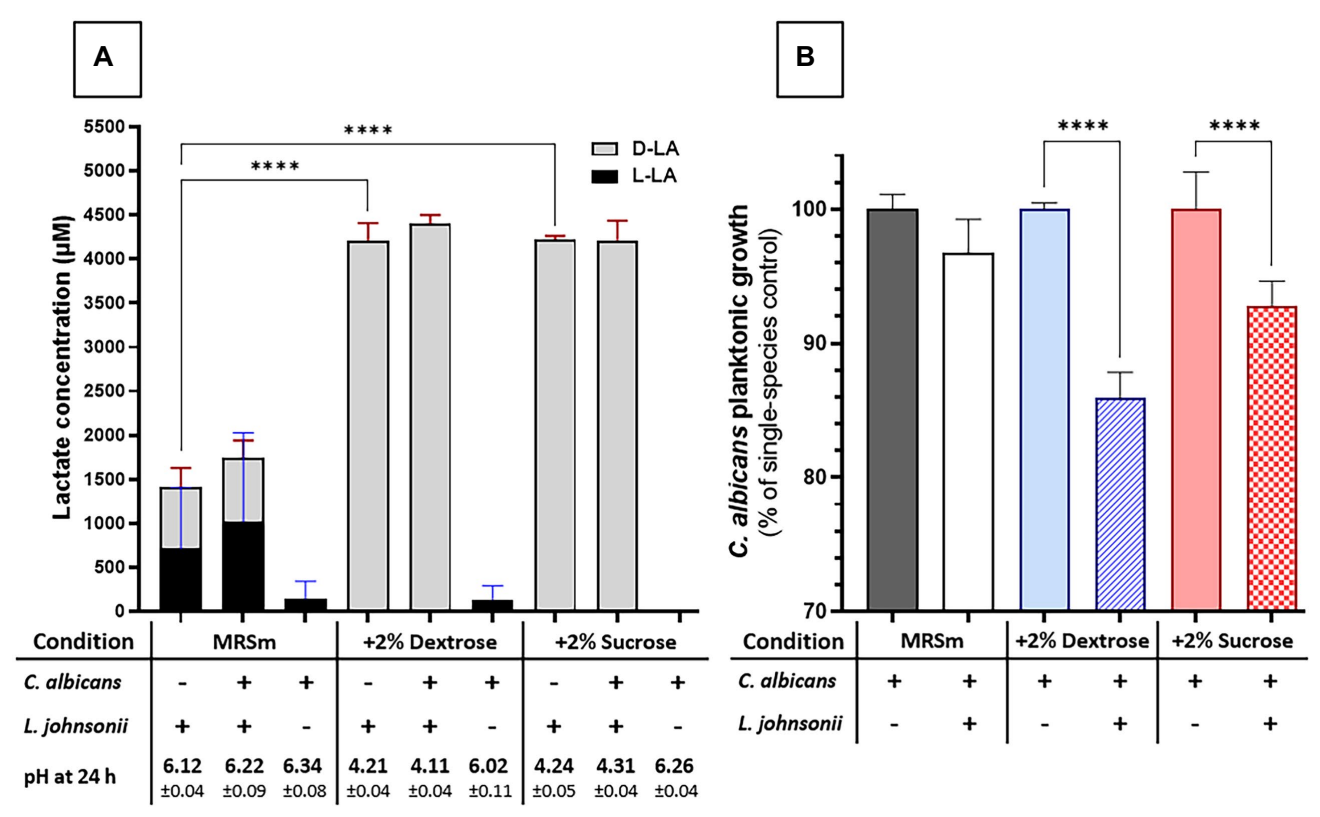

FIGURE 5 | Carbon sources influence L. johnsonii anticandidal activity, lactic acid production, and pH. (A) Lactate production is impacted by availability but not by the type of carbohydrate (i.e., sucrose vs. glucose). The acidification of the culture media correlates to lactate production; (B) The carbohydrate availability and its type influenced the anticandidal activity of $L$. johnsonii. One-Way ANOVA, Dunnett posttest, ${ }^{* * * * *} p \leq 0.0001 .100 \%$ growth corresponds to $6.51 \pm 0.06$ SD, $7.37 \pm 0.02$ $\mathrm{SD}$, and $6.71 \pm 0.06 \mathrm{SD}$ yeast cells $\mathrm{ml}^{-1}$ (average, log10 values) in MRSm, MRSm+2\% dextrose, and MRSm+2\% sucrose, respectively.
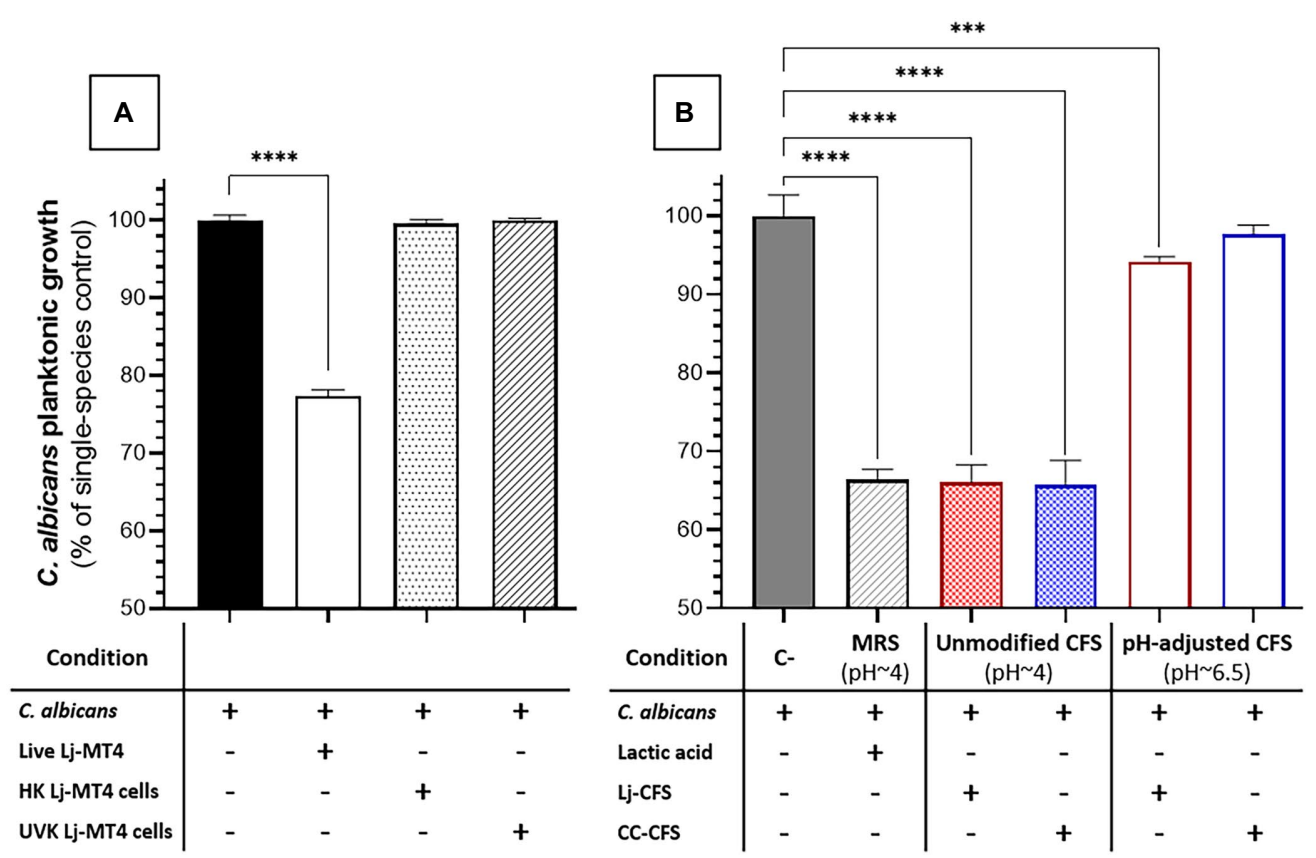

FIGURE 6 | Effect of $L$. johnsonii inactivated cells and soluble metabolites on C. albicans growth in MRS broth. (A) Inactivated $L$. johnsonii cells did not reduce the growth of $C$. albicans; (B) L. johnsonii CFS inhibited the growth of $C$. albicans in a pH-dependent manner. HK=heat killed, UVK=UV-killed. CFS $=$ Cell-free supernatant (spent media). One-Way ANOVA, Dunnett posttest, ${ }^{* * * *} p \leq 0.005,{ }^{* * * * *} p \leq 0.0001 .100 \%$ growth corresponds to $8.16 \pm 0.41 \mathrm{SD}^{*}$ yeast cells $\mathrm{ml}^{-1}$ in $\mathrm{MRS}^{*}$ broth.

of C. albicans to form biofilms was significantly hindered by the MT4 strain (Figure 7C), decreasing the fungal biofilm biovolumes (Figure 7E), thickness (Figures 7A,C,F, side view), and biomass as assessed by qPCR (Figure 7G). L. johnsonii ATCC 33200 displayed similar antibiofilm activity to strain MT4 (Figures 7D-G). In addition to hindering the formation 

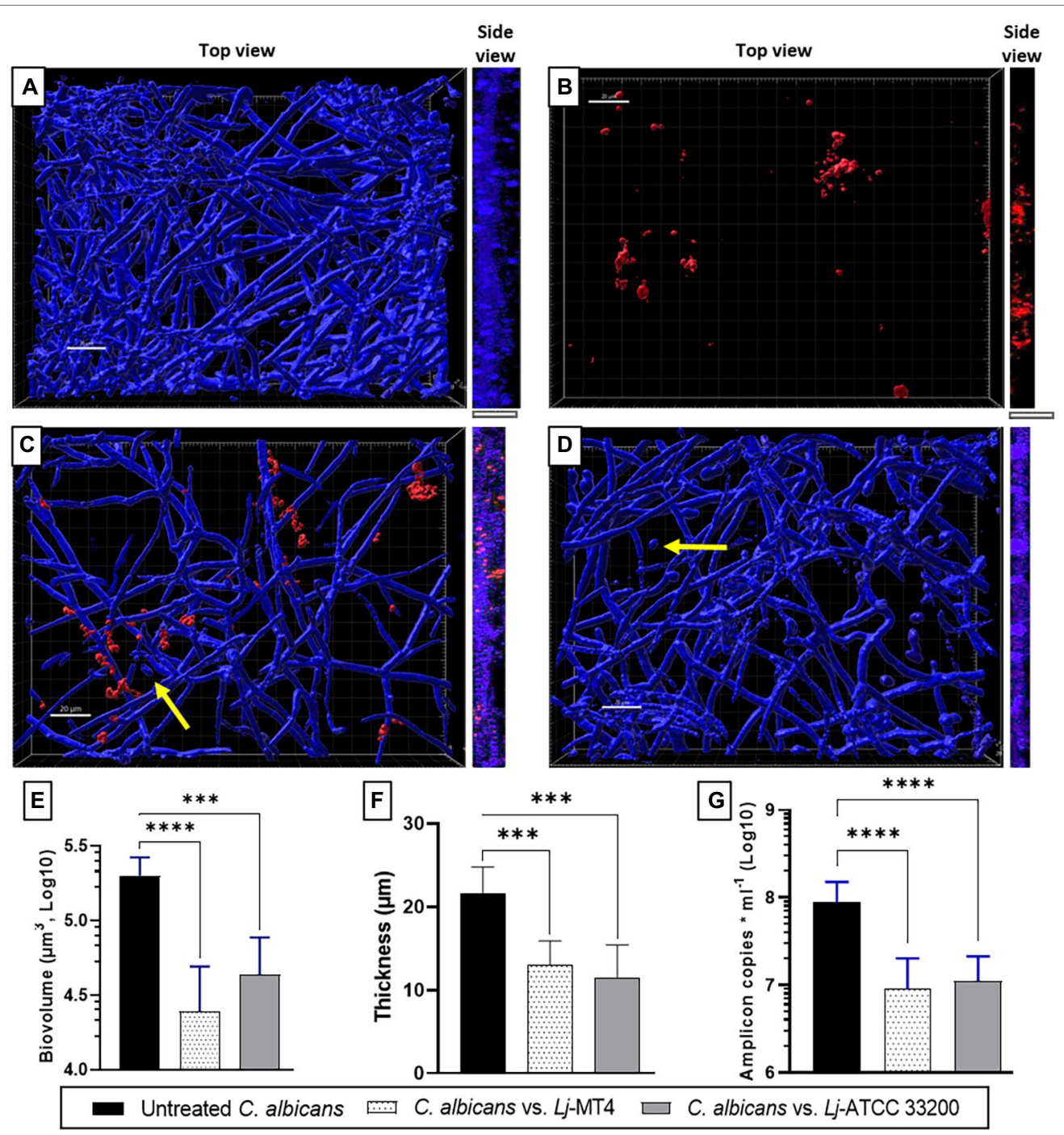

FIGURE 7 | Lactobacillus johnsonii reduces the ability of C. albicans to form biofilms. (A) Single C. albicans biofilms uniformly cover the surface; (B) Single L. johnsonii biofilms consist of scattered clusters of cells; C. albicans biofilm formation is reduced by L. johnsonii (C) MT4 and (D) ATCC 33200. Fungal biofilm (E) biovolumes, (F) thickness, and (G) biomass are significantly reduced in the presence of lactobacilli. Yellow arrows indicate the presence of yeast cells. White bar $=20 \mu$. One-Way ANOVA, Dunnett posttest. ${ }^{* * *} p \leq 0.005,{ }^{* * * *} p \leq 0.0001$.

of fungal biofilms, the yeast morphotype was present in the dual-species biofilms but was rarely observed in the singlespecies fungal biofilms. The $\mathrm{pH}$ of biofilm media at the end of single- and dual-species cultures were $6.08 \pm 0.18$ and $6.65 \pm 0.44$, respectively, suggesting that the effect of $L$. johnsonii on biofilm growth is not due to media acidification by the bacteria under these growth conditions.

To examine the role of physical contact between the two organisms in Candida biofilm growth inhibition, we used transwell inserts during biofilm growth, allowing passage of soluble metabolites from lactobacilli seeded on the upper transwell compartment. Physical separation of microorganisms with transwell inserts did not significantly impact the reduction in C. albicans biofilm biomass, showing that physical contact between L. johnsonii and C. albicans is not required for inhibiting fungal biofilm growth (Supplementary Figure 6). Instead, these data suggested that either nutrient competition during coculture or secreted L. johnsonii metabolites are responsible for this effect. These results agree with studies using a similar design that showed other Lactobacillus species having biofilm inhibitory activity against $C$. albicans (Poupet et al., 2019) and Streptococcus mutans (Wu et al., 2015) in a contact-independent way. To further explore the impact of secreted metabolites, we tested the CFS prepared from biofilm cultures on fungal biofilm growth. CFS did not reduce the fungal biofilm biovolumes compared to the 50\% PBS control (Figure 8A), but caused a small but statistically significant reduction in the metabolic activity of the fungal biofilms (Figure 8B). Importantly, CSF from L. Johnsonii alone or from coculture with C. albicans hindered its ability to transition into hyphae (Figures 8C-G). Surprisingly the yeast morphotype was the most abundant in all CFS-treated biofilms, regardless of whether the $\mathrm{pH}$ of the 

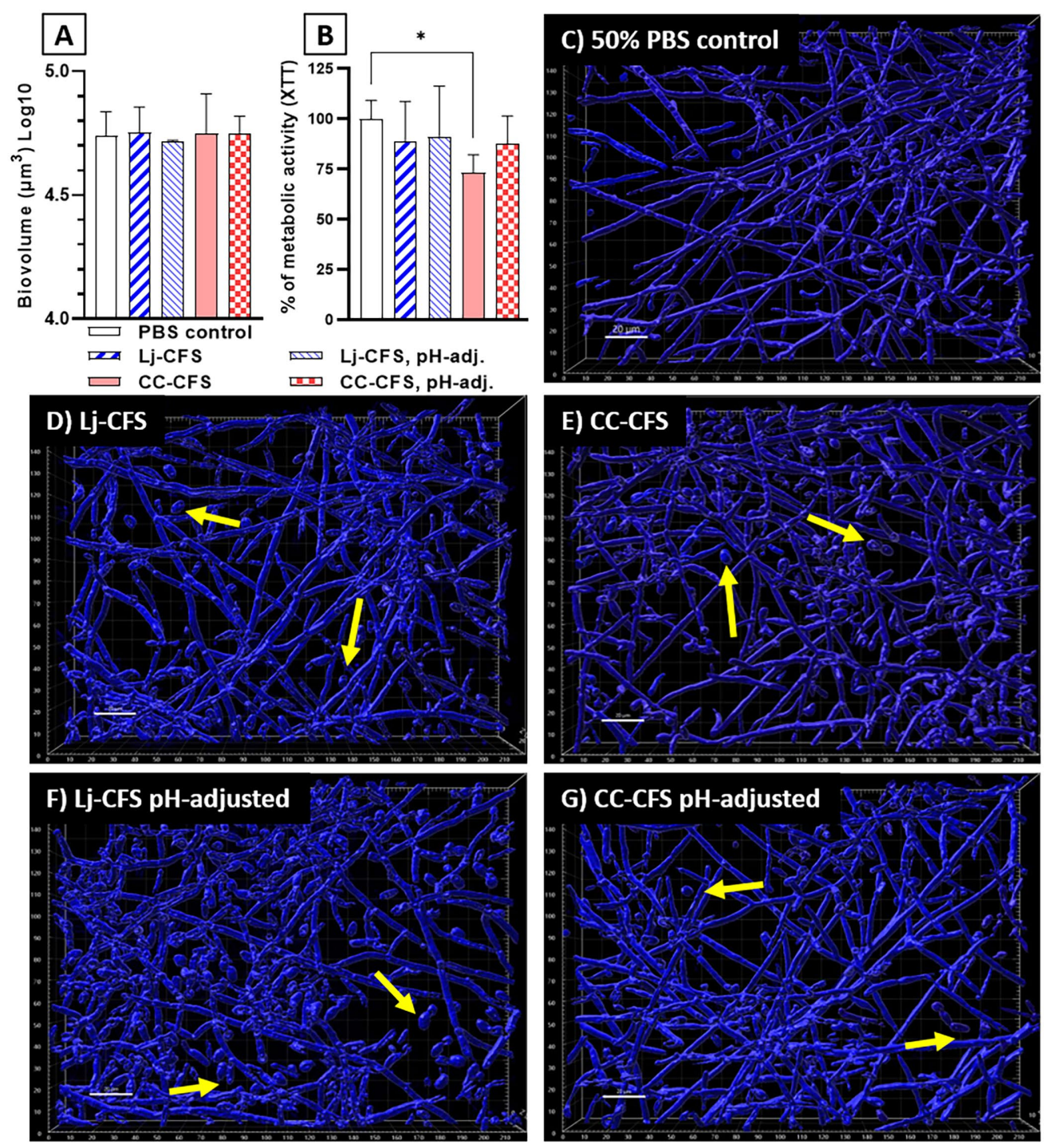

FIGURE 8 | Cell-Free Supernatants reduce the ability of $C$. albicans for hyphal transition in biofilm growth. CFS were prepared from single $L$. johnsonii biofilms (Lj-CFS, panels D,F) or from biofilm cocultures with C. albicans (CC-CFS, panels E,G) in biofilm media and were added during biofilm growth. CSF were used with and without $\mathrm{pH}$ neutralization to discern the metabolites' activity beyond acidification. CFS did not reduce the fungal biofilm biovolumes (panel A) but reduced the average metabolic activity of the biofilms (B). Additionally, all CFS supplements notably increased the yeast morphotype numbers in biofilms. (C-G). Yellow arrows indicate yeast cells. White bar $=20 \mu$. One-Way ANOVA, Dunnett posttest. ${ }^{*} p \leq 0.05 .100 \%$ of XTT metabolic activity (panel B) corresponds to OD $490=0.48 \pm 0.05$ SD of biofilms in $50 \%$ PBS.

CSF was adjusted before adding to the culture media or not (Figures 8D-G). These results, taken together, suggest that Lactobacillus metabolites, accumulating over time during growth in biofilm medium, can negatively impact fungal metabolic activity and hinder the dimorphic transition in a $\mathrm{pH}$-independent manner.

Based on these observations, we next hypothesized that $24 \mathrm{~h}$ preformed biofilms of $L$. johnsonii will reduce the ability of C. albicans to form biofilms (Figures 9A,C,E-G). Indeed there was a significant reduction in biofilm growth as confirmed with biovolume (Figure 9E), thickness (Figures 9A,C,F, side view), and biomass (Figure 9G) estimates. As expected, a large number of cells remained in the yeast morphotype, suggesting that the Lactobacillus preformed biofilm reduced the dimorphic transition into hyphae, which is an essential stage in biofilm growth on mucosal surfaces (Chandra et al., 2001). Along the same lines, when lactobacilli were added to the 24-h Candida preformed biofilms, further growth of C. albicans biofilms was prevented (Figure 9D) as seen in comparison to $48 \mathrm{~h}$ control biofilms (Figures 9A,B). Thickness and biomass estimates in this setting were similar to the initial $24 \mathrm{~h}$ biofilm control, suggesting arrested biofilm growth. However, fungal biofilm biovolumes (Figure 9E) were significantly lower than both the 24 and $48 \mathrm{~h}$ control biofilms, possibly due to the fact that lactobacilli-treated biofilms were not uniformly distributed on the surface (Figure 9D). The 

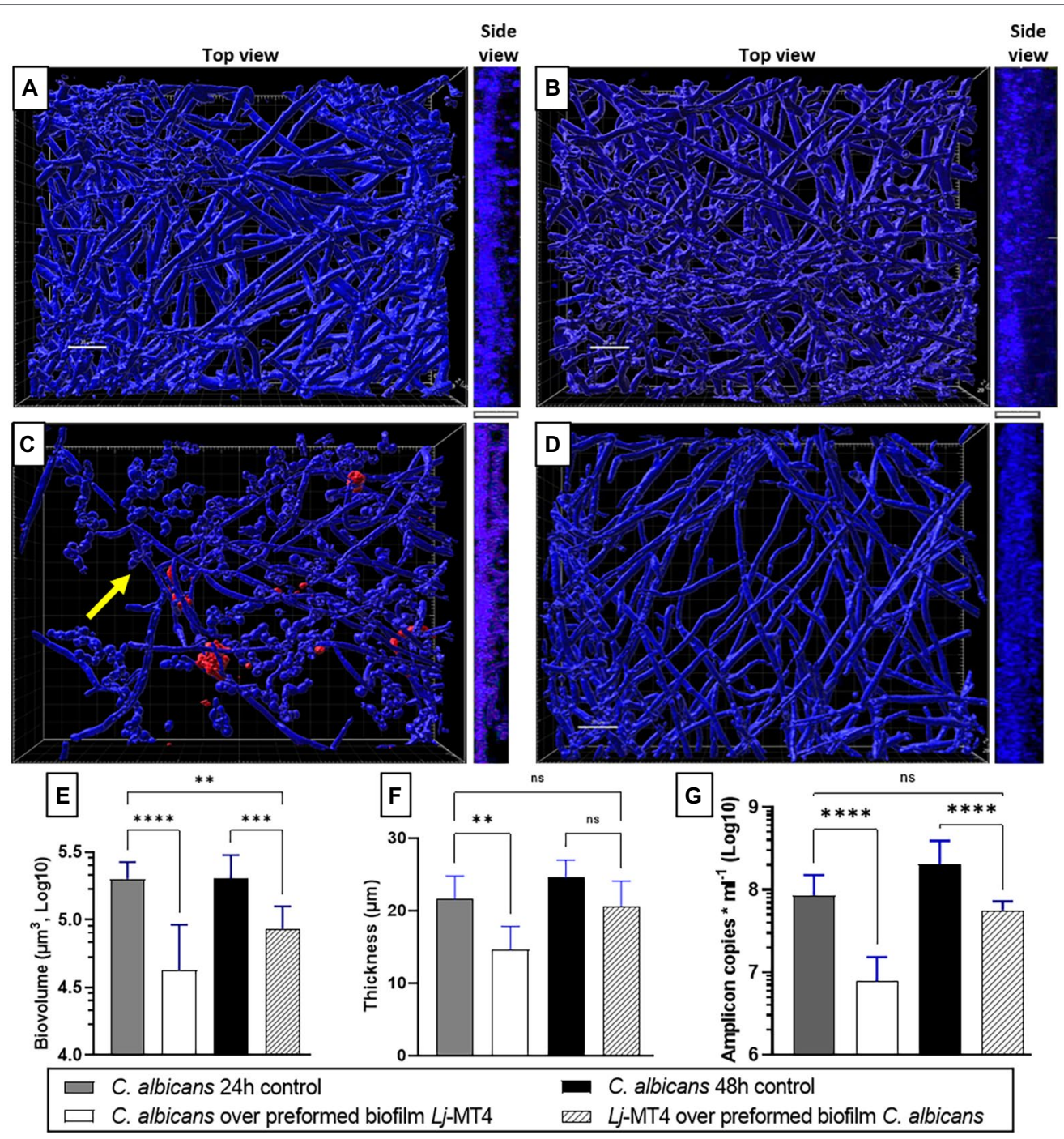

FIGURE 9 | Lactobacillus johnsonii displays exclusion and displacement effects against C. albicans biofilms. (A) 24h-old and (B) 48h-old single C. albicans biofilms; (C) Addition of C. albicans on preformed L. johnsonii biofilm; (D) Addition of L. johnsonii over preformed C. albicans biofilm; Fungal biofilm (E) biovolumes, (F) thickness, and (G) biomass are significantly reduced in the presence of lactobacilli. Yellow arrows indicate the presence of yeast cells. White bar $=20 \mathrm{~nm}$. OneWay ANOVA, Dunnett posttest. ${ }^{*} p \leq 0.05,{ }^{* * *} p \leq 0.01,{ }^{* * * *} p \leq 0.005,{ }^{* * * *} p \leq 0.0001$.

ability of L. johnsonii to alter the fungal biofilm structure and prevent its further growth indicates an antagonistic relationship between these species.

\section{DISCUSSION}

In this work, we showed that, like other Lactobacillus species (Kang et al., 2018; Jang et al., 2019; Scillato et al., 2021), L. johnsonii displays anticandidal properties, reducing C. albicans growth and ability to transition into hyphae and establish biofilms on abiotic surfaces. We found that the MT4 strain shows strong dose-dependent anticandidal activity, particularly in the biofilm growth phase. The anticandidal activity of L. johnsonii depends on several factors such as viability, cell density, nutrient availability, production of metabolites, and partly on acidification, which are all influenced by growth conditions. Genomic analysis of this strain revealed the presence of several genes that encode metabolites with anticandidal properties, which may explain our results. These metabolites are analogous to other anticandidal compounds isolated and characterized from different bacterial species (i.e., Bacillus subtilis and other members of the genus Lactobacillus). For example, Bacillomycin D (from B. subtilis) is a fungicidal lipopeptide bacteriocin from the iturin group, which targets the cell membrane, creating ion-conducting pores due to the formation of lipopeptide-sterol complexes (Olfa et al., 2015). Additionally, Bacillomycin D-like peptides inhibit $\beta$-1,3-glucan synthesis, a major component of the fungal cell wall (Hajare et al., 2016). Surfactin, produced by different Lactobacillus species, is a cyclo-lipopeptide biosurfactant that reduces substrate adhesion of C. albicans, decreasing its ability to form biofilms 
(Nelson et al., 2020). The major secreted protein Msp1 is a hydrolase that cleaves chitin, one of the main biopolymers in the fungal cell wall. Recently, this hydrolase was implicated in inhibiting C. albicans morphogenesis into hyphae (Allonsius et al., 2019) and reducing the virulence of C. glabrata in a mouse model (Charlet et al., 2020). It is possible that any or all of these metabolites are involved in the anticandidal activities we observed in vitro. In addition to these metabolites, data from other lactobacilli (Bergsson et al., 2001; Schaefer et al., 2010; Gomaa, 2013; Charlet et al., 2020) suggest that L. johnsonii may encode novel antimicrobial products that require further characterization.

The role of lactate/lactic acid and media acidification on $C$. albicans has been widely discussed in the literature, sometimes with opposite conclusions. Köhler et al. showed that C. albicans growth was reduced in lactic acid-supplemented MRS; however, when the supplemented MRS was neutralized, Candida resumed its expected growth, suggesting that acidification and not lactate is the leading cause of growth inhibition (Köhler et al., 2012). In contrast, Lourenço et al. showed that C. albicans growth is not significantly reduced in lactic acid-supplemented minimal media under acidic conditions (Lourenço et al., 2019), implying that acidification is not relevant under the tested conditions. Our results show that the impact of acidification on C. albicans growth inhibition depends on the culture media, as acidification played a key role on MRS broth, but not in BHI or biofilm medium, which did not acidify significantly during co-culture. Also, the D-/L-lactate ratio was different across the growth media, with D-lactate the enantiomer primarily produced in MRS and the $\mathrm{L}$ enantiomer in $\mathrm{BHI}$ and biofilm medium. Candida albicans metabolizes L-lactate produced by the microbiota and the host, but cannot process D-lactate produced only by the microbiota. The ability of $C$. albicans to metabolize L-lactate produced in $\mathrm{BHI}$ and biofilm medium and neutralize the $\mathrm{pH}$ may be associated with the higher $\mathrm{pH}$ we observed in CandidaLactobacillus co-cultures in these media compared to single cultures or cocultures in MRS (Danhof et al., 2016). Beyond its impact on fungal growth, lactic acid/lactate influence fungal physiology and morphology. Exposure for $76 \mathrm{~h}$ or longer to lactic acid, and other weak organic acids, turns the yeast cells into a "starvationlike" state, with slow growth rates and RNA-associated metabolism (Cottier et al., 2015). Additionally, lactate reduces the biosynthesis of ergosterol and induces incorrect localization of the transporter Cdr1, reducing the efflux of fluconazole in C. albicans cells (Suchodolski et al., 2021), leading to improving the efficacy of azoles, in combined treatments, against C. albicans (Alves et al., 2017; Lourenço et al., 2019). Disruption of ergosterol synthesis may interfere with fungal susceptibility to Bacillomycin D, which forms complexes with ergosterol, causing pores on the cell membrane. On the other hand, lactate also contributes to masking C. albicans $\beta$-glucans (Ene et al., 2013), allowing the fungi to elude the immune system. Yet, $\beta$-glucan masking is triggered by L-lactate but not by its D isomer (Ballou et al., 2016). The enantiomer produced by lactobacilli colonizing mucosal sites in vivo is unknown, but our results in the serum-supplemented biofilm media suggest that the L-enantiomer may be more physiologically relevant and thus likely to play a significant role in fungal recognition by innate immune cells and the overall host-microbiota-Candida interactions.

Lactobacillus johnsonii MT4 displayed an auto-aggregation phenotype. While not all strains of L. johnsonii display this phenotype (Jankovic et al., 2003), aggregation is a relevant trait in several ecological niches, including within the host mucosal sites, as it promotes the interaction between microbial cells. Similarly, as suggested for other L. johnsonii strains (Gil et al., 2010), MT4 can co-aggregate with C. albicans. In the context of the oral cavity, co-aggregation is a physical interaction mechanism that positively influences colonization and biofilm formation (Kolenbrander, 2000).

We observed that preformed biofilms of lactobacilli significantly inhibited fungal biofilms and the yeast's ability to shift to hyphae. These properties of the mouse MT4 strain may explain the dominant yeast phenotype of $C$. albicans on the oral mucosa of mice receiving a sucrose-rich diet which promotes the growth of lactobacilli (Souza et al., 2020; Bertolini et al., 2021). In addition to preventing Candida from forming biofilms, L. johnsonii MT4 disrupted preformed fungal biofilms, implying that these bacteria may also curtail further growth of biofilms in colonized surfaces. Quorum sensing (QS) regulates C. albicans yeast-to-hyphal transition and biofilm formation (Kruppa, 2009). Diverse QS molecules (i.e., farnesol and fatty acids) can prevent yeast from shifting into hyphae (Lee et al., 2021). Lactobacilli can inhibit QS-induced biofilms in bacteria (Aman et al., 2021), but their impact on $C$. albicans QS responses requires further investigation.

In conclusion, we showed that L. johnsonii has an antagonistic relationship with $C$. albicans during planktonic and biofilm growth in vitro. Environmental variables, such as the type and amount of nutrients, influence L. johnsonii MT4 metabolism, and anticandidal activity. Genomic analysis revealed that beyond acidification and lactate, other soluble metabolites may be responsible for the anticandidal activity and are the focus of current and future investigations. Our findings suggest that this species displays promising probiotic properties to prevent or treat mucosal candidiasis.

\section{DATA AVAILABILITY STATEMENT}

The raw FASTQ sequencing data are available at the NCBI Sequence Read Archive under accession SRR17309641. The assembled MT4 genome sequence was submitted to the NCBI Genome database with accession JAJQJG000000000. The MT4 BioSample SAMN23838460 and other associated data listed previously are available under BioProject PRJNA787656.

\section{AUTHOR CONTRIBUTIONS}

RV-M: experimental work, analysis, and writing. AT: resources and experimental work. JR: genomic analysis and repositories deposition. TS: resources. YZ: supervision and genomic analysis. AD-B: project administration, funding acquisition, supervision, and writing. All authors contributed to the article and approved the submitted version. 


\section{FUNDING}

This study was funded by NIH/NIDCR grant RO1 DE013986 and NIH/NIGMS grant RO1 GM127909.

\section{ACKNOWLEDGMENTS}

The authors acknowledge the Center for Genome Innovation, Institute for Systems Genomics, University of Connecticut, for the

\section{REFERENCES}

Allonsius, C. N., Vandenheuvel, D., Oerlemans, E. F. M., Petrova, M. I., Donders, G. G. G., Cos, P., et al. (2019). Inhibition of Candida albicans morphogenesis by chitinase from lactobacillus rhamnosus GG. Sci. Rep. 9:2900. doi: 10.1038/s41598-019-39625-0

Altschul, S. F., Gish, W., Miller, W., Myers, E. W., and Lipman, D. J. (1990). Basic local alignment search tool. J. Mol. Biol. 215, 403-410. doi: 10.1016/ S0022-2836(05)80360-2

Alves, R., Mota, S., Silva, S., Rodrigues, C. F., Brown, A. J. P., Henriques, M., et al. (2017). The carboxylic acid transporters Jen1 and Jen2 affect the architecture and fluconazole susceptibility of Candida albicans biofilm in the presence of lactate. Biofouling 33, 943-954. doi: 10.1080/08927014. 2017.1392514

Aman, M., Aneeqha, N., Bristi, K., Deeksha, J., Afza, N., Sindhuja, V., et al. (2021). Lactic acid bacteria inhibits quorum sensing and biofilm formation of Pseudomonas aeruginosa strain JUPG01 isolated from rancid butter. C. Agric. Biotechnol. 36:102115. doi: 10.1016/J.BCAB.2021.102115

Assefa, S., Ahles, K., Bigelow, S., Curtis, J. T., and Köhler, G. A. (2015). Lactobacilli with probiotic potential in the prairie vole (Microtus ochrogaster). Gut Pathog. 7:35. doi: 10.1186/s13099-015-0082-0

Ballou, E. R., Avelar, G. M., Childers, D. S., Mackie, J., Bain, J. M., Wagener, J., et al. (2016). Lactate signalling regulates fungal $\beta$-glucan masking and immune evasion. Nat. Microbiol. 2:16238. doi: 10.1038/NMICROBIOL. 2016.238

Bankevich, A., Nurk, S., Antipov, D., Gurevich, A. A., Dvorkin, M., Kulikov, A. S., et al. (2012). SPAdes: A new genome assembly algorithm and its applications to single-cell sequencing. J. Comput. Biol. 19, 455-477. doi: 10.1089/CMB. 2012.0021

Bergsson, G., Arnfinnsson, J., Steingrímsson, Ó., and Thormar, H. (2001). In vitro killing of Candida albicans by fatty acids and monoglycerides. Antimicrob. Agents Chemother. 45, 3209-3212. doi: 10.1128/AAC.45.11.3209-3212.2001

Bertolini, M., Vazquez Munoz, R., Archambault, L., Shah, S., Souza, J. G. S., Costa, R. C., et al. (2021). Mucosal bacteria modulate Candida albicans virulence in Oropharyngeal candidiasis. mBio 12:e0193721. doi: 10.1128/mBio.01937-21

Castresana, J. (2000). Selection of conserved blocks from multiple alignments for their use in phylogenetic analysis. Mol. Biol. Evol. 17, 540-552. doi: 10.1093/oxfordjournals.molbev.a026334

Centers for Disease Control and Prevention (2019). Antibiotic Resistance Threats in the United States, 2019. Atlanta, GA. Available at: www.cdc.gov/ DrugResistance/Biggest-Threats.html (Accessed January 24, 2020).

Chandra, J., Kuhn, D. M., Mukherjee, P. K., Hoyer, L. L., McCormick, T., and Ghannoum, M. A. (2001). Biofilm formation by the fungal pathogen Candida albicans: development, architecture, and drug resistance. J. Bacteriol. 183, 5385-5394. doi: 10.1128/JB.183.18.5385-5394.2001/ASSET/7682BA161CB1-4757-B4AC-81291C643CA8/ASSETS/GRAPHIC/JB1810569007. JPEG

Charlet, R., Bortolus, C., Sendid, B., and Jawhara, S. (2020). Bacteroides thetaiotaomicron and lactobacillus johnsonii modulate intestinal inflammation and eliminate fungi via enzymatic hydrolysis of the fungal cell wall. Sci. Rep. 10:11510. doi: 10.1038/s41598-020-68214-9

Cottier, F., Tan, A. S. M., Chen, J., Lum, J., Zolezzi, F., Poidinger, M., et al. (2015). The transcriptional stress response of Candida albicans to weak organic acids. G3 5, 497-505. doi: 10.1534/G3.114.015941 whole genome sequencing of the L. johnsonii MT4 strain and CCAM Microscopy Facility, University of Connecticut Health Center, for the access to their quantitative fluorescence imaging applications.

\section{SUPPLEMENTARY MATERIAL}

The Supplementary Material for this article can be found online at: https://www.frontiersin.org/articles/10.3389/fmicb.2022.853762/ full\#supplementary-material

Cruchet, S., Obregon, M. C., Salazar, G., Diaz, E., and Gotteland, M. (2003) Effect of the ingestion of a dietary product containing lactobacillus johnsonii La1 on helicobacter pylori colonization in children. Nutrition 19, 716-721. doi: 10.1016/S0899-9007(03)00109-6

Danhof, H. A., Vylkova, S., Vesely, E. M., Ford, A. E., Gonzalez-Garay, M., and Lorenz, M. C. (2016). Robust extracellular pH modulation by Candida albicans during growth in carboxylic acids. mbio 7, e01646-e01616. doi: 10.1128/MBIO.01646-16

Dongari-Bagtzoglou, A., Kashleva, H., Dwivedi, P., Diaz, P., and Vasilakos, J. (2009). Characterization of mucosal Candida albicans biofilms. PLoS One 4:e7967. doi: 10.1371/journal.pone.0007967

Ene, I. V., Cheng, S. C., Netea, M. G., and Brown, A. J. P. (2013). Growth of Candida albicans cells on the physiologically relevant carbon source lactate affects their recognition and phagocytosis by immune cells. Infect. Immun. 81, 238-248. doi: 10.1128/IAI.01092-12

Eryilmaz, M., Gurpinar, S. S., Palabiyik, I. M., Guriz, H., and Gerceker, D. (2019). Molecular identification and antimicrobial activity of vaginal lactobacillus sp. Curr. Pharm. Biotechnol. 19, 1241-1247. doi: 10.217 4/1389201020666190110164123

Felsenstein, J. (1993). PHYLIP (Phylogeny Inference Package) version 3.57c. Available at: http://www.dbbm.fiocruz.br/molbiol/main.html (Accessed December 23, 2021).

Fujisawa, T., Benno, Y., Yaeshima, T., and Mitsuoka, T. (1992). Taxonomic study of the Lactobacillus acidophilus group, with recognition of Lactobacillus gallinarum sp. nov. and Lactobacillus johnsonii sp. nov. and synonymy of Lactobacillus acidophilus group A3 (Johnson et al. 1980) with the type strain of Lactobacillus amylovorus (Nakamura 1981). Int. J. Syst. Bacteriol. 42, 487-491. doi: 10.1099/00207713-42-3-487

Gascuel, O. (1997). BIONJ: an improved version of the NJ algorithm based on a simple model of sequence data. Mol. Biol. Evol. 14, 685-695. doi: 10.1093/oxfordjournals.molbev.a025808

Gil, N. F., Martinez, R. C. R., Gomes, B. C., Nomizo, A., and de Martinis, E. C. P. (2010). Vaginal lactobacilli as potential probiotics against Candida spp Brazilian J. Microbiol. 41, 6-14. doi: 10.1590/S1517-83822010000100002

Gomaa, E. Z. (2013). Antimicrobial and anti-adhesive properties of biosurfactant produced by lactobacilli isolates, biofilm formation and aggregation ability. J. Gen. Appl. Microbiol. 59, 425-436. doi: 10.2323/jgam.59.425

Gouy, M., Guindon, S., and Gascuel, O. (2010). SeaView version 4: A multiplatform graphical user Interface for sequence alignment and phylogenetic tree building. Mol. Biol. Evol. 27, 221-224. doi: 10.1093/molbev/msp259

Guindon, S., Dufayard, J.-F., Lefort, V., Anisimova, M., Hordijk, W., and Gascuel, O. (2010). New algorithms and methods to estimate maximumlikelihood phylogenies: assessing the performance of PhyML 3.0. Syst. Biol. 59, 307-321. doi: 10.1093/sysbio/syq010

Hajare, S. N., Gautam, S., and Sharma, A. (2016). A novel strain of Bacillus amyloliquefaciens displaying broad spectrum antifungal activity and its underlying mechanism. Ann. Microbiol. 66, 407-416. doi: 10.1007/ s13213-015-1123-0

Jang, S. J., Lee, K., Kwon, B., You, H. J., and Ko, G. P. (2019). Vaginal lactobacilli inhibit growth and hyphae formation of Candida albicans. Sci. Rep. 9, 8121-8129. doi: 10.1038/s41598-019-44579-4

Jankovic, I., Ventura, M., Meylan, V., Rouvet, M., Elli, M., and Zink, R. (2003). Contribution of aggregation-promoting factor to maintenance of cell shape in Lactobacillus gasseri 4B2. J. Bacteriol. 185, 3288-3296. doi: 10.1128/ JB.185.11.3288-3296.2003 
Jung, M. Y. (2018). Complete genome sequence of Lactobacillus sp. CBA3606. EMBL/GenBank/DDBJ. Available at: https://www.uniprot.org/uniprot/ A0A2R3JZ49 [Accessed December 24 2021].

Kang, C., Kim, Y. G., Han, S. H., Kim, J. S., Paek, N. S., and So, J. S. (2018). In vitro probiotic properties of vaginal Lactobacillus fermentum MG901 and Lactobacillus plantarum MG989 against Candida albicans. Eur. J. Obstet. Gynecol. Reprod. Biol. 228, 232-237. doi: 10.1016/j.ejogrb.2018.07.005

Khot, P. D., Ko, D. L., and Fredricks, D. N. (2009). Sequencing and analysis of fungal rRNA operons for development of broad-range fungal PCR assays. Appl. Environ. Microbiol. 75, 1559-1565. doi: 10.1128/AEM.02383-08

Köhler, G. A., Assefa, S., and Reid, G. (2012). Probiotic interference of Lactobacillus rhamnosus GR-1 and Lactobacillus reuteri RC-14 with the opportunistic fungal pathogen Candida albicans. Infect. Dis. Obstet. Gynecol. 2012, 1-14. doi: $10.1155 / 2012 / 636474$

Kolenbrander, P. E. (2000). Oral microbial communities: biofilms, interactions, and genetic systems. Annu. Rev. Microbiol. 54, 413-437. doi: 10.1146/annurev.micro.54.1.413

Kruppa, M. (2009). Quorum sensing and Candida albicans. Mycoses 52, 1-10. doi: $10.1111 /$ J.1439-0507.2008.01626.X

Lee, J. H., Kim, Y. G., Khadke, S. K., and Lee, J. (2021). Antibiofilm and antifungal activities of medium-chain fatty acids against Candida albicans via mimicking of the quorum-sensing molecule farnesol. Microb. Biotechnol. 14, 1353-1366. doi: 10.1111/1751-7915.13710

Lefort, V., Longueville, J. E., and Gascuel, O. (2017). SMS: smart model selection in PhyML. Mol. Biol. Evol. 34, 2422-2424. doi: 10.1093/MOLBEV/MSX149

Lourenço, A., Pedro, N. A., Salazar, S. B., and Mira, N. P. (2019). Effect of acetic acid and lactic acid at low $\mathrm{pH}$ in growth and azole resistance of Candida albicans and Candida glabrata. Front. Microbiol. 9:3265. doi: 10.3389/fmicb.2018.03265

Löytynoja, A. (2014). "Phylogeny-aware alignment with PRANK", in Multiple Sequence Alignment Methods. Methods in Molecular Biology (Methods and Protocols). Vol. 1079. ed. D. Russell (Totowa, NJ: Humana Press), 155-170.

Marcial, G. E., Ford, A. L., Haller, M. J., Gezan, S. A., Harrison, N. A., Cai, D., et al. (2017). Lactobacillus johnsonii N6.2 modulates the host immune responses: A double-blind, randomized trial in healthy adults. Front. Immunol. 8:655. doi: 10.3389/FIMMU.2017.00655

Mundula, T., Ricci, F., Barbetta, B., Baccini, M., and Amedei, A. (2019). Effect of probiotics on oral candidiasis: a systematic review and meta-analysis. Nutrients 11:2449. doi: 10.3390/nu11102449

Nelson, J., El-Gendy, A. O., Mansy, M. S., Ramadan, M. A., and Aziz, R. K. (2020). The biosurfactants iturin, lichenysin and surfactin, from vaginally isolated lactobacilli, prevent biofilm formation by pathogenic Candida. FEMS Microbiol. Lett. 367:126. doi: 10.1093/FEMSLE/FNAA126

O’Flaherty, S., Foley, M. H., Rivera, A. J., Theriot, C. M., and Barrangou, R. (2020). Complete genome sequence of Lactobacillus johnsonii NCK2677, isolated from mice. Microbiol. Resour. Announc. 9, e00918-e00920. doi: 10.1128/MRA.00918-20

Olfa, T., Antonio, D. G., Sana, A., Imen, B. S., Salem, E., Mohamed Najib, A., et al. (2015). Synergistic fungicidal activity of the lipopeptide bacillomycin D with amphotericin B against pathogenic Candida species. FEMS Yeast Res. 15:fov022. doi: 10.1093/femsyr/fov022

Page, A. J., Cummins, C. A., Hunt, M., Wong, V. K., Reuter, S., Holden, M. T. G., et al. (2015). Roary: rapid large-scale prokaryote pan genome analysis. Bioinformatics 31, 3691-3693. doi: 10.1093/BIOINFORMATICS/BTV421

Pappas, P. G., Lionakis, M. S., Arendrup, M. C., Ostrosky-Zeichner, L., and Kullberg, B. J. (2018). Invasive candidiasis. Nat. Rev. Dis. Prim. 4, 1-20. doi: $10.1038 /$ nrdp.2018.26

Pendleton, K. M., Dickson, R. P., Newton, D. W., Hoffman, T. C., Yanik, G. A., and Huffnagle, G. B. (2018). Respiratory tract colonization by Candida species portends worse outcomes in Immunocompromised patients. Clin. Pulm. Med. 25, 197-201. doi: 10.1097/CPM.0000000000000279

Pfaller, M. A., and Diekema, D. J. (2010). Epidemiology of invasive mycoses in North America. Crit. Rev. Microbiol. 36, 1-53. doi: 10.3109/10408410903241444

Pfaller, M. A., Diekema, D. J., Turnidge, J. D., Castanheira, M., and Jones, R. N. (2019). Twenty years of the SENTRY antifungal surveillance program: results for Candida species from 1997-2016. Open Forum Infect. Dis. 6, S79-S94. doi: $10.1093 /$ ofid/ofy358

Pierce, C. G., Uppuluri, P., Tristan, A. R., Wormley, F. L., Mowat, E., Ramage, G., et al. (2008). A simple and reproducible 96-well plate-based method for the formation of fungal biofilms and its application to antifungal susceptibility testing. Nat. Protoc. 3, 1494-1500. doi: 10.1038/nprot.2008.141
Poupet, C., Saraoui, T., Veisseire, P., Bonnet, M., Dausset, C., Gachinat, M., et al. (2019). Lactobacillus rhamnosus Lcr35 as an effective treatment for preventing Candida albicans infection in the invertebrate model Caenorhabditis elegans: first mechanistic insights. PLoS One 14:e0216184. doi: 10.1371/journal.pone.0216184

Schaefer, L., Auchtung, T. A., Hermans, K. E., Whitehead, D., Borhan, B., and Britton, R. A. (2010). The antimicrobial compound reuterin (3-hydroxypropionaldehyde) induces oxidative stress via interaction with thiol groups. Microbiology 156, 1589-1599. doi: 10.1099/mic.0.035642-0

Scillato, M., Spitale, A., Mongelli, G., Privitera, G. F., Mangano, K., Cianci, A., et al. (2021). Antimicrobial properties of Lactobacillus cell-free supernatants against multidrug-resistant urogenital pathogens. Microbiology 10:e1173. doi: $10.1002 / \mathrm{mbo3} .1173$

Seemann, T. (2014). Prokka: rapid prokaryotic genome annotation. Bioinformatics 30, 2068-2069. doi: 10.1093/BIOINFORMATICS/BTU153

Souza, J. G. S., Bertolini, M., Thompson, A., Mansfield, J. M., Grassmann, A. A., Maas, K., et al. (2020). Role of glucosyltransferase $\mathrm{R}$ in biofilm interactions between Streptococcus oralis and Candida albicans. ISME J. 14, 1207-1222. doi: 10.1038/s41396-020-0608-4

Strus, M., Kucharska, A., Kukla, G., Brzychczy-Włoch, M., Maresz, K., and Heczko, P. B. (2005). The in vitro activity of vaginal Lactobacillus with probiotic properties against Candida. Infect. Dis. Obstet. Gynecol. 13, 69-75. doi: $10.1080 / 10647440400028136$

Suchodolski, J., Muraszko, J., Bernat, P., and Krasowska, A. (2021). Lactate like fluconazole reduces ergosterol content in the plasma membrane and synergistically kills Candida albicans. Int. J. Mol. Sci. 22:5219. doi: 10.3390/ IJMS22105219

Vazquez-Munoz, R., Arellano-Jimenez, M. J., and Lopez-Ribot, J. L. (2020). Bismuth nanoparticles obtained by a facile synthesis method exhibit antimicrobial activity against Staphylococcus aureus and Candida albicans. BMC Biomed. Eng. 2:11. doi: 10.1186/s42490-020-00044-2

Vazquez-Munoz, R., and Dongari-Bagtzoglou, A. (2021). Anticandidal activities by lactobacillus species: An update on mechanisms of action. Front. Oral Heal. 2:689382. doi: 10.3389/froh.2021.689382

Wagner, R. D., Pierson, C., Warner, T., Dohnalek, M., Hilty, M., and Balish, E. (2000). Probiotic effects of feeding heat-killed lactobacillus acidophilus and lactobacillus casei to Candida albicans-colonized immunodeficient mice. J. Food Prot. 63, 638-644. doi: 10.4315/0362-028x-63.5.638

Wood, D. E., Lu, J., and Langmead, B. (2019). Improved metagenomic analysis with Kraken 2. Genome Biol. 20:257. doi: 10.1186/S13059-019-1891-0

Wu, C. C., Lin, C. T., Wu, C. Y., Peng, W. S., Lee, M. J., and Tsai, Y. C. (2015). Inhibitory effect of lactobacillus salivarius on Streptococcus mutans biofilm formation. Mol Oral Microbiol 30, 16-26. doi: 10.1111/OMI.12063

Yamano, T., Tanida, M., Niijima, A., Maeda, K., Okumura, N., Fukushima, Y., et al. (2006). Effects of the probiotic strain lactobacillus johnsonii strain La1 on autonomic nerves and blood glucose in rats. Life Sci. 79, 1963-1967. doi: $10.1016 /$ J.LFS.2006.06.038

Zheng, J., Wittouck, S., Salvetti, E., Franz, C. M. A. P., Harris, H. M. B., Mattarelli, P., et al. (2020). A taxonomic note on the genus lactobacillus: description of 23 novel genera, emended description of the genus lactobacillus beijerinck 1901, and union of Lactobacillaceae and Leuconostocaceae. Int. J. Syst. Evol. Microbiol. 70, 2782-2858. doi: 10.1099/ijsem.0.004107

Conflict of Interest: The authors declare that the research was conducted in the absence of any commercial or financial relationships that could be construed as a potential conflict of interest.

Publisher's Note: All claims expressed in this article are solely those of the authors and do not necessarily represent those of their affiliated organizations, or those of the publisher, the editors and the reviewers. Any product that may be evaluated in this article, or claim that may be made by its manufacturer, is not guaranteed or endorsed by the publisher.

Copyright $\odot 2022$ Vazquez-Munoz, Thompson, Russell, Sobue, Zhou and DongariBagtzoglou. This is an open-access article distributed under the terms of the Creative Commons Attribution License (CC BY). The use, distribution or reproduction in other forums is permitted, provided the original author(s) and the copyright owner(s) are credited and that the original publication in this journal is cited, in accordance with accepted academic practice. No use, distribution or reproduction is permitted which does not comply with these terms. 